\title{
Evaluation of surgical outcomes of obstetric brachial plexus birth injury: clinical and electrophysiological study Diaa F. Mohasseb ${ }^{a}$, Marwa M. Hassan ${ }^{a}$, Ahmed E. Semaya ${ }^{b}$, Hayam M. Abdelghany ${ }^{\mathrm{c}}$, Sarah N. Hassan ${ }^{\mathrm{d}}$
}

Departments of aPhysical Medicine, Rheumatology and Rehabilitation, ${ }^{\text {b } O r t h o p e d i c ~}$ Surgery, Faculty of Medicine, Alexandria University, ${ }^{\circ}$ Department of Physical Medicine, Rheumatology and Rehabilitation, Alexandria University Hospitals, Alexandria, 'Department of Physical Medicine, Rheumatology and Rehabilitation, Faculty of Medicine, Helwan University, Helwan, Egypt

Correspondence to Marwa M. Hassan, MD, Department of Physical Medicine,

Rheumatology and Rehabilitation, Faculty of Medicine, Alexandria University, Alexandria, 21111, Egypt. Tel: +20 100277 3457; fax: 034847426;

e-mail: drmarwamohamedhassan@yahoo.com

Received 17 January 2019

Accepted 3 April 2019

Egyptian Rheumatology \& Rehabilitation 2019, 46:229-236

\begin{abstract}
Background
Obstetric brachial plexus birth injury (OBPBI) is a relatively common form of birth injury that might lead to a weighty physical disability. Early microsurgical intervention for properly selected patients will result in maximal functional benefit that couldn't be otherwise obtained. However, the outcomes of different microsurgical techniques need to be further evaluated and compared.

Aim

To evaluate the outcomes of different microsurgical techniques performed in patients with OBPBI.

\section{Patients \& Methods}

Twenty-eight patients with OBPBI meeting the criteria for surgical intervention were included in the study. Preoperative and one year postoperative clinical and electrophysiological assessments were done.

Results

Thirteen (46.4\%) of the studied patients were males and 15 (53.6\%) were females. The mean age of patients in the study was 8.64 months \pm 4.40 ; ranging between (3.0 - 16.0 months), 7 patients had Horner syndrome.C5 and C6+/-C7 roots affection was the most frequent lesion ,patients who underwent neurotization recovered earlier than patients who underwent nerve grafting ,on the other hand nerve grafting resulted in more significant postoperative improvement after a 12 months follow up period.

\section{Conclusions}

The current study provided additional evidence that the earlier the surgical intervention in OBPBI, the better the outcomes.Neurotization results in earlier functional recovery in patients with $\mathrm{OBPBI}$ than nerve grafting reconstructive techniques. On the other hand, nerve grafting was superior to neurotization regarding the degree of improvement. Postoperative electrophysiological followup can be done using the CMAP amplitude and AUC percentages reflecting the percentage of viable axons.
\end{abstract}

\section{Keywords:}

electrophysiological assessment, microsurgical techniques, obstetric brachial plexus birth injury

Egypt Rheumatol Rehabil 46:229-236

(C) 2019 Egyptian Society for Rheumatology and Rehabilitation

$1110-161 X$

\section{Introduction}

Obstetric brachial plexus birth injury (OBPBI) is a relatively harmful form of birth injury that might lead to significant physical disability.

Detailed history and clinical examination, together with imaging and electrodiagnostic tools, allow localization of the level and severity of the injury to the brachial plexus. This allows prognostication of recovery and a decision to be made for surgery [1].

A commonly used assessment tool is the active movement scale (AMS) of the hospital for sick children [2]. Fifteen different movements in the affected arms are assessed and scored on an eightpoint scale. The infant is enticed to move using a variety of play stimuli. The eight gradations allow for discrimination of movements. It allows for comparison of preoperative and postoperative results.

Most brachial plexus lesions are axonal in nature. The recorded amplitude and negative area under the curve (AUC) of the compound muscle action potential (CMAP), which reflect the total number of conducting fibers, are decreased. In contrast, the latencies and conduction velocities $(\mathrm{CVs})$ reflect the conduction rate along only the fastest conducting fibers (i.e. those with the largest diameters and the thickest myelin sheaths), thus only reflect a small percentage of

\footnotetext{
This is an open access journal, and articles are distributed under the terms of the Creative Commons Attribution-NonCommercial-ShareAlike 4.0 License, which allows others to remix, tweak, and build upon the work non-commercially, as long as appropriate credit is given and the new creations are licensed under the identical terms.
} 
the stimulated nerve fibers. Consequently, these values do not become abnormal until all or most of the fastest fibers are affected [3].

Therefore, even with severe axon loss lesions demonstrating low CMAP amplitudes, whenever some of the fastest fibers remain unaffected, latency and CV values remain normal. The amplitude and negative AUC values are by far the most-sensitive nerve conduction study (NCS) parameters for identifying axon loss lesions, whereas the latency and $\mathrm{CV}$ values are fairly insensitive until the lesion is quite severe [4]. Accordingly, the motor responses are extremely helpful in severity assessment and pathophysiology determination. Specifically, the amplitude and negative AUC values can be used to estimate the percentage of affected motor axons and the underlying pathophysiology [3].

It is agreed that in those children who meet the criteria for surgical intervention, treatment should not be delayed, as this is likely to prejudice the maximal benefit that could otherwise be obtained [5].

The decision of early surgical intervention is straightforward in case of an infant with a flail limb and an associated Horner syndrome [6]. Moreover, failure of spontaneous return of elbow flexion at 3 months of age is the most widely accepted criteria for surgical intervention in infants with an upper plexus lesion [7].

The most widely used microsurgical techniques for OBPBI are neurolysis, neurotization, and nerve grafting [8].

There remains controversy as to whether NCSs and needle electromyography (EMG) are helpful in predicting recovery or selecting patients for surgical intervention $[9,10]$.

The outcomes of different reconstructive microsurgical procedures need to be assessed and compared using clinical, functional, and electrophysiological tools for proper selection and prediction of surgical outcomes in patients with OBPBI.

The aim of this work was to evaluate the outcomes of different surgical techniques performed in patients with OBPBI.

\section{Patients}

Twenty-eight infants with OBPBI meeting the criteria for surgical intervention $[6,7]$ were enrolled in this study.
The most important and agreed upon recommendation to perform primary reconstructive surgery is the lack of biceps function at 3-6 months of age [6].

Patients were excluded if they have any neurological disorders that can cause weakness of the upper limb [11].

All patients' parents were informed about the nature of the study, and an informed consent was taken before the beginning of the study, which was approved by the local ethical committee of Faculty of Medicine, Alexandria University.

\section{Methods}

All studied patients were subjected to preoperative and 1-year postoperative clinical and electrophysiological assessments.

\section{Clinical assessment}

Apart from the routine neurological examination of the affected upper limb, AMS was used for functional assessment [2]. Fifteen cardinal movements of the upper extremity were assessed, including those of the shoulder (abduction, adduction, flexion, internal rotation, and external rotation), elbow (flexion and extension), forearm (pronation and supination), and wrist, fingers, and thumb (flexion and extension). Each movement is assessed and graded on an eight-point scale, and each grade is converted into a score (Table 1 ).

\section{Electrophysiological assessment}

Electrophysiological procedures were carried out using Neuropack S1EMG/EP Measuring System (Nihon Kohden, Tokyo, Japan). All recorded action potentials were carried out by surface electrodes $(8 \mathrm{~mm})$ in diameter, and a ground electrode was placed between the stimulating and recording electrodes. The filter setting was between 2 and $10 \mathrm{~Hz}$ [12]. Sensory conduction study was

Table 1 The hospital for sick children active movement scale converted scores [2]

\begin{tabular}{lcc}
\hline Observation & Muscle grade & Converted score \\
\hline Gravity eliminated & & \\
No contraction & 0 & 0 \\
Contraction, no motion & 1 & 0.3 \\
Motion $<1 / 2$ range & 2 & 0.3 \\
Motion $>1 / 2$ range & 3 & 0.6 \\
Full motion & 4 & 0.6 \\
Against gravity & & \\
Motion $<1 / 2$ range & 5 & 0.6 \\
Motion $>1 / 2$ range & 6 & 1.3 \\
Full motion & 7 & 2 \\
\hline
\end{tabular}


performed for median and ulnar nerve, whereas motor conduction study was done for median, ulnar, radial (from wrist extensors and from triceps), musculocutaneous, axillary, and suprascapular nerves [12]. The studied parameters were latency, amplitude, $\mathrm{CV}$ (if applicable), and AUC of the recorded response.

Sensory conduction studies of the median and ulnar nerves were done to localize the level of the injury and to determine whether it is preganglionic or postganglionic.

The percentage of viable axons was calculated by using the CMAP amplitude (CMAP amplitude of the affected side/CMAP amplitude of the contralateral sidex 100) and by using AUC (AUC of the affected side/AUC of the contralateral side $\times 100)$.

\section{Surgical intervention}

The anterior approach to the brachial plexus was used in all cases, because it allows access to the entire plexus from roots to peripheral nerves. Standard microsurgical techniques were employed. The magnification used during dissection and graft harvesting or preparation of other donor nerves ranged from loupes $\times 2.5$ to $\times 4$.

After exploration of the brachial plexus, the surgeon decision for the type of operation is made, either reconstruction by nerve grafting or neurotization. If the decision is reconstructive procedure using nerve grafts, harvesting of one or both sural nerves and medial cutaneous nerve of the arm was performed. If neurotization was the option, it was done by using either intercostal nerves to musculocutaneous nerve or spinal accessory nerve to suprascapular nerve.

\section{Statistical analysis of the data}

Data were fed to the computer and analyzed using IBM SPSS software package, version 20.0. (IBM Corp., Armonk, New York, USA). Qualitative data were described using number and percent. The Kolmogorov-Smirnov test was used to verify the normality of distribution. Quantitative data were described using range (minimum and maximum), mean, SD, and median. Significance of the obtained results was judged at the 5\% level. To compare quantitative variables between two studied groups, Student's $t$ test was used for normally distributed data and Mann-Whitney test for abnormally distributed data. Wilcoxon's signed ranks test was used for abnormally distributed quantitative variables, to compare between two periods.

\section{Results}

\section{Demographic and clinical data}

Thirteen (46.4\%) of the studied patients were males and $15(53.6 \%)$ were females. The mean age of patients in the study was $8.64 \pm 4.40$ months, ranging between 3.0 and 16.0 months. The right upper limb was affected in $16(57.1 \%)$ patients and the left upper limb was affected in 12 (42.9\%) patients. Regarding the maternal age, the mean age of the mothers in the study was 31.29 \pm 4.37 years, ranging between 21.0 and 38.0 years. Twenty-five (89.3\%) patients were delivered by normal vaginal delivery, and only three (10.7\%) patients were delivered by cesarean section. The demographic and clinical data are outlined in Table 2.

\section{Surgical data}

The most frequently detected pathological pattern intraoperatively was affection of all roots, that is C5-T1. The least detected pattern was affection of $\mathrm{C} 5$ and $\mathrm{C} 6$ roots as shown in Table 3.

Regarding the surgical procedures, neurotization was done in 13 (46.4\%) patients and reconstruction using nerve grafting was done in 15 (53.6\%) patients.

Table 2 Distribution of the studied cases according to demographic and clinical data $(N=28)$

\begin{tabular}{|c|c|}
\hline & $n(\%)$ \\
\hline \multicolumn{2}{|l|}{ Sex } \\
\hline Male & $13(46.4)$ \\
\hline Female & $15(53.6)$ \\
\hline \multicolumn{2}{|c|}{ Age at assessment and intervention (months) } \\
\hline Minimum-maximum & $3.0-16.0$ \\
\hline Mean $\pm S D$ & $8.64 \pm 4.40$ \\
\hline Median & 6.50 \\
\hline \multicolumn{2}{|l|}{ Affected side } \\
\hline Right & $16(57.1)$ \\
\hline Left & $12(42.9)$ \\
\hline \multicolumn{2}{|l|}{ Maternal age (years) } \\
\hline Minimum-maximum & $21.0-38.0$ \\
\hline Mean \pm SD & $31.29 \pm 4.37$ \\
\hline Median & 32.0 \\
\hline \multicolumn{2}{|l|}{ Mode of delivery } \\
\hline Cesarean section & $3(10.7)$ \\
\hline Normal vaginal & $25(89.3)$ \\
\hline \multicolumn{2}{|l|}{ Fetal presentation } \\
\hline Cephalic & $28(100.0)$ \\
\hline Prolonged labor & $16(57.1)$ \\
\hline Shoulder dystocia & $16(57.1)$ \\
\hline Gestational DM & $16(57.1)$ \\
\hline Multiparty & $23(82.1)$ \\
\hline Macrosomia & $21(75.0)$ \\
\hline Previous infant with Erb's & $2(7.1)$ \\
\hline \multicolumn{2}{|l|}{ Horner syndrome } \\
\hline Absent & $21(75.0)$ \\
\hline Present & $7(25.0)$ \\
\hline
\end{tabular}

DM, diabetes mellitus. 


\section{Clinical follow-up data}

The mean of preoperative AMS converted score was $5.53 \pm 5.17$, whereas the postoperative mean was 10.71 \pm 3.87 (Table 4).

There was a statistically significant difference between the AMS overall converted score presurgically and postsurgically $(P<0.001)$, that is, the studied patients' overall upper limb function was significantly improved after surgical intervention as shown in Table 4.

Table 3 Distribution of the studied patients according to the intraoperative pathology $(\mathrm{N}=\mathbf{2 8})$

\begin{tabular}{lc}
\hline Intraoperative findings & $n(\%)$ \\
\hline C5 to T1 affection & $12(42.9)$ \\
C5 and C6 affection & $6(21.4)$ \\
C5, C6, and C7 affection & $10(35.7)$ \\
\hline
\end{tabular}

There was a statistically significant difference between the two studied surgical groups regarding the AMS improvement percentage, that is, patients who underwent reconstruction by nerve grafting improved more significantly compared with the patients who underwent neurotization $(P=0.019)$, as shown in Table 5 .

Regarding the onset of functional recovery, there was a statistically significant difference between the two studied surgical groups, that is, patients who underwent neurotization recovered earlier than those who underwent reconstruction by nerve grafting $(P=0.048)$, as shown in Table 6.

\section{Electrophysiological follow-up data}

Electrophysiological improvement percentage was calculated for each nerve. The musculocutaneous

Table 4 Comparison between the overall functional active movement scale converted scores preoperatively and postoperatively $(N=28)$

\begin{tabular}{lccc}
\hline Score & Preoperative & Postoperative & $Z$ \\
\hline Total score & & & \\
$\quad$ Minimum-maximum & $0.0-16.50$ & $4.80-18.50$ & \\
Mean \pm SD & $5.53 \pm 5.17$ & $10.71 \pm 3.87$ & $4.441^{*}$ \\
Median & 4.50 & 9.40 & \\
\hline
\end{tabular}

$Z$, Wilcoxon signed-rank test. $P, P$ value for comparing between before and after. ${ }^{*}$ Statistically significant at $P$ value less than or equal to 0.05 .

Table 5 Comparison between the two studied surgical groups according to improvement percentage in active movement scale total score

\begin{tabular}{|c|c|c|c|c|c|}
\hline \multirow[t]{2}{*}{ AMS total score } & \multirow[t]{2}{*}{ Total $(N=28)$} & \multicolumn{2}{|c|}{ Surgical procedure } & \multirow[t]{2}{*}{$U$} & \multirow[t]{2}{*}{$P$} \\
\hline & & Neurotization $(N=13)$ & Nerve grafting $(N=15)$ & & \\
\hline \multicolumn{6}{|l|}{ Preoperative } \\
\hline Minimum-maximum & $0.0-16.50$ & $1.50-15.30$ & $0.0-16.50$ & & \\
\hline Mean \pm SD & $5.43 \pm 5.01$ & $7.72 \pm 4.34$ & $3.45 \pm 4.82$ & $37.5^{\star}$ & $0.004^{*}$ \\
\hline Median & 4.50 & 6.30 & 1.80 & & \\
\hline \multicolumn{6}{|l|}{ Postoperative } \\
\hline Minimum-maximum & $4.80-18.50$ & $4.80-18.50$ & $7.60-18.20$ & & \\
\hline Mean $\pm S D$ & $10.81 \pm 3.92$ & $11.91 \pm 4.69$ & $9.86 \pm 2.93$ & 68.0 & 0.185 \\
\hline Median & 9.40 & 12.20 & 8.70 & & \\
\hline \multicolumn{6}{|l|}{ Improvement percentage } \\
\hline Minimum-maximum & $0.60-8.70$ & $0.60-8.50$ & $1.70-8.70$ & & \\
\hline Mean \pm SD & $5.38 \pm 2.46$ & $4.19 \pm 2.28$ & $6.41 \pm 2.20$ & $45.50^{\star}$ & $0.015^{\star}$ \\
\hline Median & 5.75 & 4.50 & 7.50 & & \\
\hline
\end{tabular}

AMS, active movement scale; $U$, Mann-Whitney test. $P, P$ value for comparing between neurotization and nerve grafting. ${ }^{\star}$ Statistically significant at $P$ value less than or equal to 0.05 .

Table 6 Comparison between the two studied surgical groups according to the onset of functional recovery (months)

\begin{tabular}{lcc}
\hline & \multicolumn{2}{c}{ Surgical procedure } \\
Onset of early recovery (months) & Neurotization MSC (ICN) $(N=13)$ & Nerve graft $(N=15)$ \\
\hline Minimum-maximum & $4.0-10.0$ & $7.0-12.0$ \\
Mean \pm SD & $7.92 \pm 2.06$ & $9.47 \pm 1.88$ \\
Median & 8.0 & 9.0 \\
\hline
\end{tabular}

ICN, intercostal nerve; MSC, musculocutaneous nerve. $t$, Student $t$ test. $P, P$ value for comparing between neurotization and nerve graft. *Statistically significant at $P$ value less than or equal to 0.05 . 
nerve had the highest improvement percentage detected by CMAP amplitude and AUC (37.53 \pm 30.17 and $33.44 \pm 24.35$, respectively), and the least improvement percentage was in the suprascapular nerve $(24.86 \pm 30.30$ and $7.66 \pm 47.62$, respectively), as shown in Tables 7 and 8 (Figs 1 and 2).

\section{Discussion}

OBPBI is a significant form of birth injury that might lead to significant physical and psychological disability. Although Egyptian statistics about the frequency of OBPBI are lacking, but a worldwide incidence of 0.5 to $3 / 1000$ birth still is considered inevitable $[13,14]$.

This work primarily aimed at evaluating the outcomes of different surgical techniques performed in patients with OBPBI.

In the current study, 13 (46.4\%) of the studied patients were males and $15(53.6 \%)$ were females; this is in

Table 7 Improvement percentage according to the amplitude of compound muscle action potential

\begin{tabular}{lccc}
\hline Nerves (roots) & Preoperative AMP (\%) & Postoperative AMP (\%) & Improvement (\%) \\
\hline Musculocutaneous & $29.64 \pm 26.21$ & $67.17 \pm 41.05$ & $37.53 \pm 30.17$ \\
Axillary & $31.30 \pm 26.65$ & $67.12 \pm 29.61$ & $35.82 \pm 15.76$ \\
Radial-TR & $19.48 \pm 32.58$ & $51.76 \pm 32.44$ & $32.28 \pm 23.65$ \\
Radial-El & $20.01 \pm 35.47$ & $47.76 \pm 39.68$ & $27.75 \pm 30.95$ \\
Suprascapular & $15.04 \pm 29.13$ & $39.90 \pm 26.05$ & $24.86 \pm 30.30$ \\
\hline
\end{tabular}

AMP, amplitude. Data were expressed using mean \pm SD. Radial-El, recording from extensor indices; radial-TR, recording from triceps.

Table 8 Improvement percentage according to the area under the curve of compound muscle action potential

\begin{tabular}{lccc}
\hline Nerves (roots) & Preoperative AUC (\%) & Postoperative AUC (\%) & Improvement (\%) \\
\hline Musculocutaneous & $33.67 \pm 24.25$ & $67.11 \pm 30.42$ & $33.44 \pm 24.35$ \\
Axillary & $37.72 \pm 27.12$ & $67.72 \pm 24.71$ & $30.0 \pm 35.10$ \\
Radial-EI & $17.53 \pm 26.60$ & $43.66 \pm 33.82$ & $26.12 \pm 34.24$ \\
Radial-TR & $35.67 \pm 30.77$ & $54.77 \pm 25.32$ & $19.10 \pm 21.32$ \\
Suprascapular & $28.39 \pm 52.97$ & $36.05 \pm 21.79$ & $7.66 \pm 47.62$ \\
\hline
\end{tabular}

Data were expressed using mean \pm SD. AUC, area under the curve; radial-El, recording from extensor indices; radial-TR, recording from triceps.

Figure 1

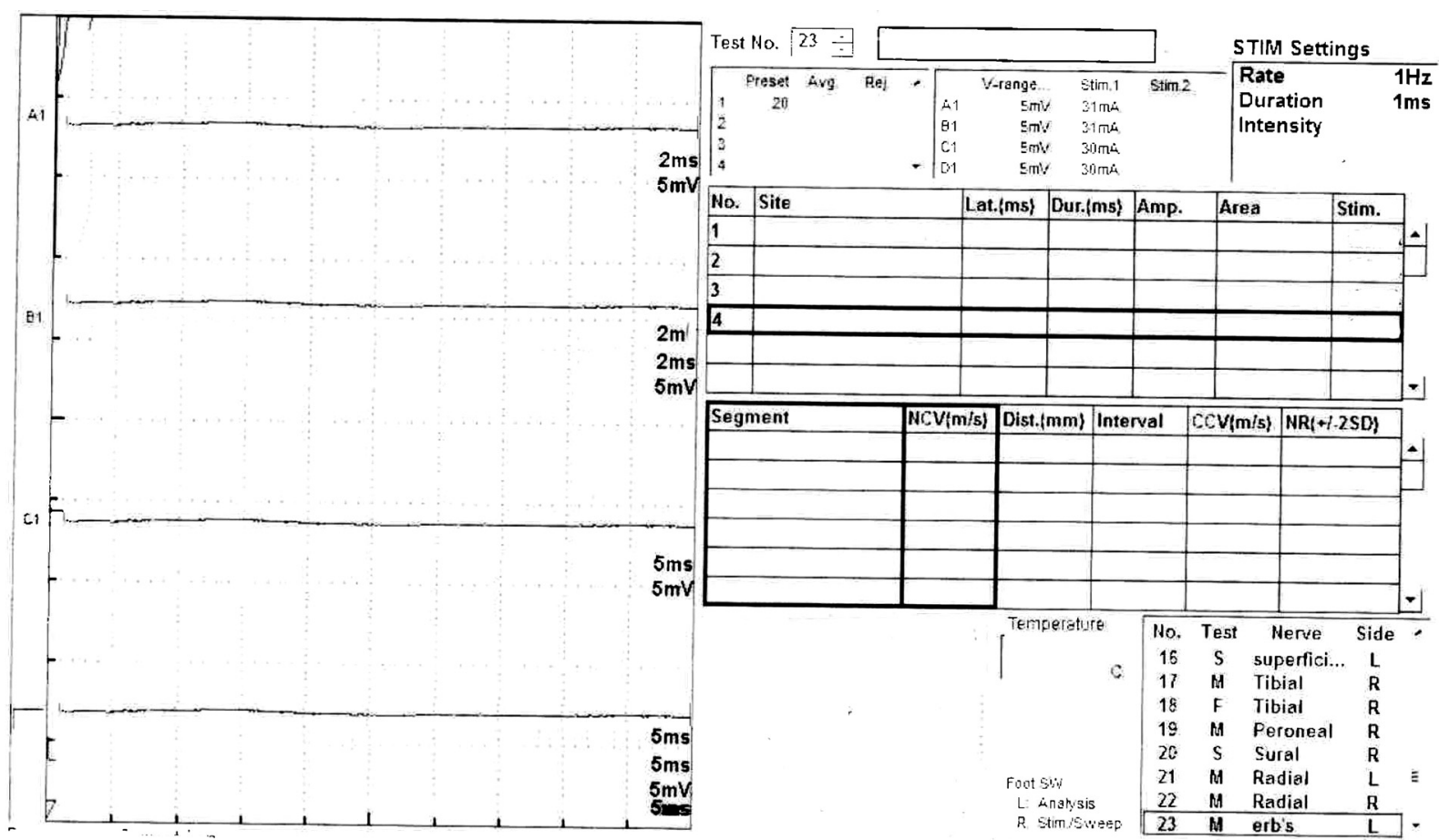

Preoperative electrophysiological assessment (proximal, motor) of a 7-month-old girl with left extended Erb's palsy $(\mathrm{C} 5,6$, and 7) and ruptured suprascapular nerve showing absent CMAP of all the studied nerves. CMAP, compound muscle action potential. 
Figure 2
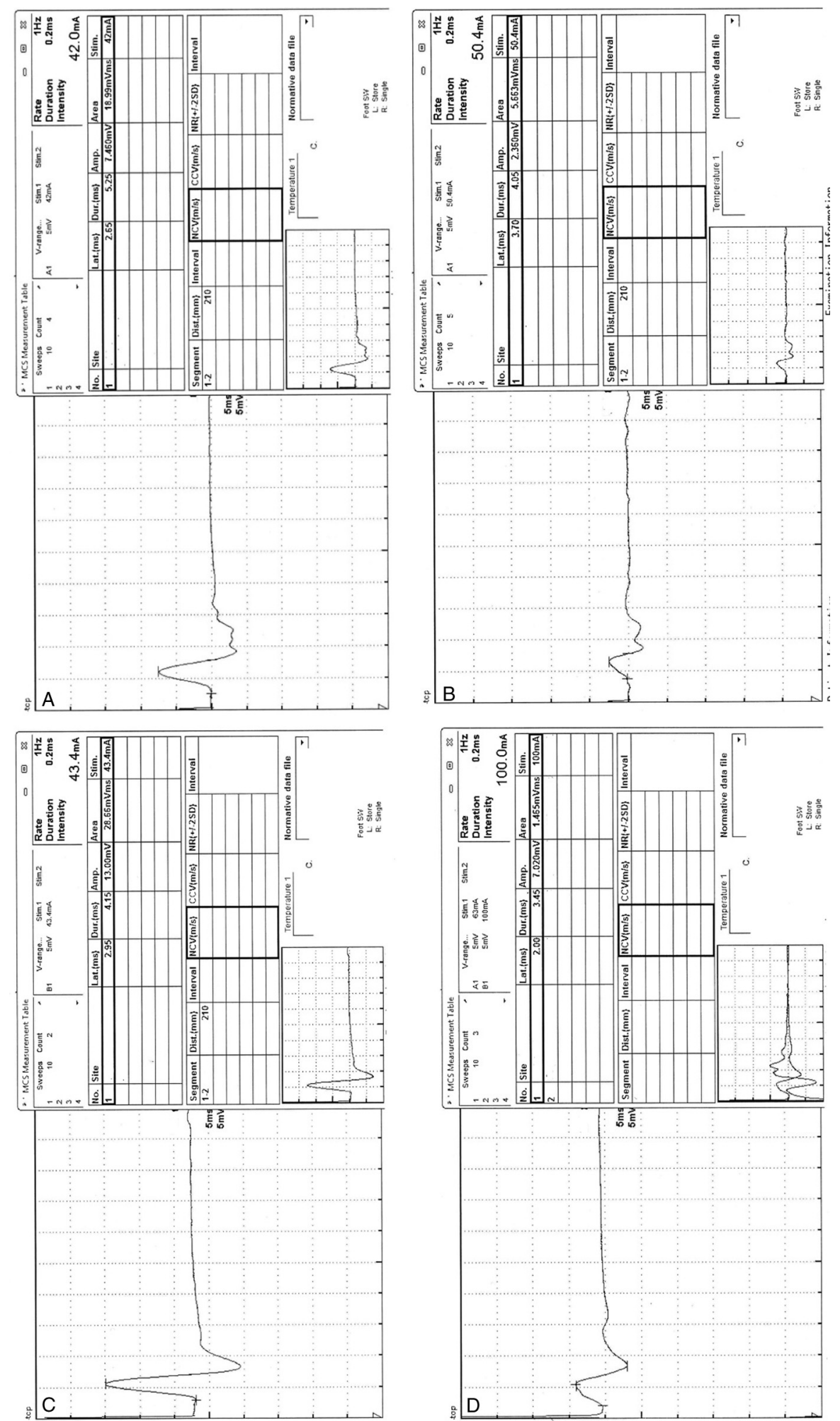

(a) One-year postoperative (reconstruction by sural nerve grafting) electrophysiological assessment of the same patient showing improvement of all conduction parameters (DL, amplitude, and AUC) of musculocutaneous nerve. (b) One-year postoperative (reconstruction by sural nerve grafting) electrophysiological assessment of the same patient showing improvement of all conduction parameters (DL, amplitude, and AUC) of the radial nerve. (c) One-year postoperative (reconstruction by sural nerve grafting) electrophysiological assessment of the same patient showing improvement of all conduction parameters (DL, amplitude, and AUC) of the axillary nerve. (d) One-year postoperative (reconstruction by sural nerve grafting) electrophysiological assessment of the same patient showing improvement of all conduction parameters (DL, amplitude, and $A \cup C$ ) of the suprascapular nerve. AUC, area under the curve. 
agreement with what Michelow et al. [15] had reported about equal sex incidence of OBPBI.

Mean age of assessment and surgical intervention in the current study was $8.64 \pm 4.40$ months, although this is considered relatively late for early microsurgical intervention, which is agreed upon by many authors to be at $3-6$ months $[6,7]$. This may be explained by the lack of appropriate health education, so parents may seek medical consultation late, resulting in late referral of cases and hence later surgical intervention, in addition to the fact that in our community considerable number of pregnant females still give birth at home without medical supervision and proper neonatal assessment.

Three $(10.7 \%)$ of the patients in the current study were delivered by cesarean section whereas 25 (89.3\%) patients were delivered normally. McFarland et al. [16] were the first to note that cesarean section did not totally eliminate the risk for OBPBI. The right upper limb was affected in 16 (57.1\%) patients and the left upper limb was affected in 12 (42.9\%) patients. In a study that included 24 infants with OBPBI, Al Shishtawy [17] found that there was more affection in the right side as three only were left sided and 21 were right sided.

Surgical decision and planning should be tailored for each patient with OBPBI depending on several factors. The most widely used technique for restoring function after OBPBI is neuroma resection and nerve grafting. However, neurotization technique is gaining increased popularity as an addition to nerve grafting or as a solo procedure [18].

Nerve grafting has been the mainstay of surgical treatment in patients with OBPBI for many years. However, increasing use of neurotization in reconstructing traumatic brachial plexus injuries in adults has led to better outcomes in relation to nerve grafting in some lesions [19], and hence, reports relating to neurotization in OBPBI cases became more frequent $[20,21]$.

In our study, patients who underwent reconstruction using nerve grafting improved more significantly (measured by their overall AMS scores) compared with the patients who underwent neurotization. This might be attributed to the fact that the decision of nerve grafting in most of our cases was made early owing to the presence of flail upper limb or absent active elbow flexion at 3-6 months of age, and most surgical opinions agree that further delay is not advisable, as poorer results should be expected, especially regarding hand function [22], which could explain better outcomes in our series of patients who underwent nerve grafting procedure.

On the contrary, and in regards to the onset of functional recovery, patients who underwent neurotization recovered earlier than those who underwent reconstruction by nerve grafting. This is consistent with a recently published series comparing outcomes after grafting and neurotization in patients with extended Erb's palsy, which demonstrated that nerve transfers provide faster recovery, but that there was no difference in outcomes after 24 months after surgery [23].

Many authors agree that neurotization do have an important role to play on certain occasions, including failed primary grafting reconstruction, late presentation, and isolated deficits [18].

The amplitude and negative AUC values are by far the most-sensitive NCS parameters for identifying axon loss lesions, whereas the latency and $\mathrm{CV}$ values are fairly insensitive until the lesion is quite severe [4].

The negative AUC is the surface area located under the negative phase of the motor response. It can be calculated by multiplying the amplitude $(\mathrm{mV})$ by the negative phase duration (in $\mathrm{ms}$ ) to yield the area $(\mathrm{mV}-\mathrm{ms})$. Like the amplitude, the negative AUC reflects the number of innervated muscle fibers. Thus, it is directly proportional to the number of conducting motor nerve fibers. Because it reflects all of the conducting motor nerve fibers (rather than the most synchronous ones), it is slightly more accurate than the amplitude for approximating the number of conducting motor nerve fibers. Like the amplitude value, the value of the negative AUC is proportional to the number of innervated muscle fibers [4].

Because it reflects all of the innervated muscle fibers, AUC is somewhat more accurate for severity estimation than is the amplitude value, and it also can be compared with the expected value (i.e. the value recorded from the contralateral asymptomatic extremity or to the control value) to estimate the percentage of nonconducting motor nerve fibers. In such way, we calculated the electrophysiological improvement percentage using the CMAP amplitude and AUC and found that the musculocutaneous nerve (reflecting mainly $\mathrm{C} 6$ root) was the most improved among all the studied nerve roots.

The results from microsurgical reconstruction vary, and comparison between different series is almost impossible, given the differences in preoperative 
evaluation, surgical strategies, and outcome analysis. As a consequence of this difficulty, the results from microsurgical treatment in the literature are quite variable $[18,24]$.

Although the current study assessed the patients clinically, functionally, and electrophysiologically 1year postoperatively, the relatively small number of the studied patients could be considered as a limitation of the current work. So, it recommended to conduct further center-based research on OBPBI on larger number of patients and longer follow-up duration.

\section{Conclusion}

(1) The current study provided additional evidence that the earlier the surgical intervention in OBPBI, the better the outcomes.

(2) Neurotization results in earlier functional recovery in patients with OBPBI than nerve grafting reconstructive techniques. On the contrary, nerve grafting was superior to neurotization regarding the degree of improvement.

(3) Postoperative electrophysiological follow-up can be done using the CMAP amplitude and AUC percentages reflecting the percentage of viable axons.

\section{Financial support and sponsorship}

Nil.

\section{Conflicts of interest}

There are no conflicts of interest.

\section{References}

1 Chim H, Bishop AT, Spinner RJ, Shin AY. Traumatic brachial plexus injury in the pediatric population. In Abzug JM, Kozin S, Zlotolow D, eds. The pediatric upper extremity. New York: Springer-Verlag 2015. 685-688

2 Curtis C, Stephens D, Clarke HM, Andrews D. The active movement scale: an evaluative tool for infants with obstetrical brachial plexus palsy. J Hand Surg Am 2002; 27:470-478.

3 Ferrante MA. Brachial plexopathies: classification, causes, and consequences. Muscle Nerve 2004; 30:547-568.
4 Ferrante MA. Electrodiagnostic assessment of the brachial plexus. Neurol Clin 2012; 30:551-580.

5 Swan MC, Clarke HM. Microsurgery for obstetrical brachial plexus palsy. In Abzug JM, Kozin SH, Zlotolow DA eds. The pediatric upper extremity. New York, NY: Springer New York 2015. 607-631

6 Al-Qattan MM. The outcome of Erb's palsy when the decision to operate is made at 4 months of age. Plast Reconstr Surg 2000; 106:1461-1465.

7 Gilbert A, Pivato G, Kheiralla T. Long-term results of primary repair of brachial plexus lesions in children. Microsurgery 2006; 26:334-342.

8 Andersen J, Watt J, Olson J, Van Aerde J. Perinatal brachial plexus palsy. Paediatr Child Health 2006; 11:93-100.

9 Heise CO, Lorenzetti L, Marchese AJ, Gherpelli JL. Motor conduction studies for prognostic assessment of obstetrical plexopathy. Muscle Nerve 2004; 30:451-455.

10 Van Dijk JG, Pondaag W, Malessy MJ. Obstetric lesions of the brachial plexus. Muscle Nerve 2001; 24:1451-1461.

11 Alfonso I, Papazian O, Grossman JA. Clinical presentations, differential diagnosis and management of obstetric brachial palsy. Rev Neurol 1998; 27:258-263.

12 Preston DC, Shapiro BE. Electromyography and neuromuscular disorders e-book: clinical-electrophysiologic correlations (expert consult-online) USA: Elsevier Health Sciences 2012.

13 Foad S, Mehlman C, Ying J. The epidemiology of neonatal brachial plexus palsy in the United States. Am J Bone Jt Surg 2008; 90:1258-1264.

14 Volpe KA, Snowden JM, Cheng YW, Caughey AB. Risk factors for brachial plexus injury in a large cohort with shoulder dystocia. Arch Gynecol Obstet 2016; 294:925-929.

15 Michelow B, Clarke $\mathrm{H}$, Curtis $\mathrm{C}$. The natural history of obstetrical brachial plexus palsy. Plast Reconstr Surg 1994; 93:675-680.

16 McFarland LV, Raskin M, Daling JR, Benedetti TJ. Erb/Duchenne's palsy: a consequence of fetal macrosomia and method of delivery. Obstet Gynecol 1986; 68:784-788

17 Al Shishtawy NI. Prediction of functional outcome of obstetric brachial plexus palsy. Egypt Rheumatol Rehab 2003; 30:625-632.

18 Siqueira M, Heise C, Alencar G, Martins R, Foroni L. Outcomes from primary surgical reconstruction of neonatal brachial plexus palsy in 104 children. Child Nerv Sys 2019; 35:349-354.

19 Garg R, Merrell GA, Hillstrom HJ, Wolfe SW. Comparison of nerve transfers and nerve grafting for traumatic upper plexus palsy: a systematic review and analysis. J Bone Joint Surg Am 2011; 93:819-829.

20 Al-Qattan MM. Oberlin's ulnar nerve transfer to the biceps nerve in Erb's birth palsy. Plast Reconstr Surg 2002; 109:405-407.

21 Noaman HH, Shiha AE, Bahm J. Oberlin's ulnar nerve transfer to the bíceps motor nerve in obstetric brachial plexus palsy: indications, and good and bad results. Microsurgery 2004; 24:182-187.

22 Chuang DC, Mardini S, Ma HS. Surgical strategy for infant obstetrical brachial plexus palsy: experiences at Chang Gung Memorial Hospital. Plast Reconstr Surg 2005; 116:132-142.

23 Heise CO, Siqueira MG, Martins RS, Foroni LH, Sterman-Neto H. Distal nerve transfer versus supraclavicular nerve grafting: comparison of elbow flexion outcome in neonatal brachial plexus palsy with $\mathrm{C} 5-\mathrm{C} 7$ involvement. Childs Nerv Syst 2017; 33:1571-1574.

24 Sarac C, Duijnisveld BJ, van der Weide A, Schoones JW, Malessy MJ, Nelissen RG, et al. Outcome measures used in clinical studies on neonatal brachial plexus palsy: a systematic literature review using the International Classification of Functioning, Disability and Health. J Pediatr Rehabil Med 2015; 8:167-185. 